\title{
TEMPERATURE AND STRAIN SENSITIVITY OF LONG PERIOD GRATING FIBER SENSOR: REVIEW
}

\author{
Liji Sreenivasan Madhavan', Manisha Chattopadhyay ${ }^{2}$ \\ ${ }^{I} P G$ student, Electronics and Telecommunication Department, VESIT, Maharashtra, India \\ ${ }^{2}$ Associate Professor, Electronics and Telecommunication Department, VESIT, Maharashtra, India
}

\begin{abstract}
Long period grating fibers are special class of fibers which are produced by periodic modulation of the refractive index of the core. This perturbation leads to forward guiding mode to couple with co propagating cladding modes depending on the phase matching condition. This coupling causes the cladding modes to attenuate during propagation in the fiber and leads to dips in the attenuation bands at discrete wavelengths in the transmission spectrum. These bands are shifted when the LPG is exposed to temperature, strain, refractive index changes in surrounding environment etc. This forms the basis of LPG sensor. The properties of LPG are modified in order to achieve the required sensitivity towards any measurands as per the applications. The performance of $L P G$ by modifying the properties of $L P G$ is reviewed.
\end{abstract}

Keywords: Optical fiber, Optical fiber sensor, Fiber Bragg, Long period grating, Strain, Temperature

\section{INTRODUCTION}

Optical fibers are devices that have the ability to transfer energy from one spatial mode to another. These optical fibers can be used as effective sensors because it can be used in remote and hazardous environments that suffer from severe electromagnetic interference. Optical fiber grating has made significant contributions in research and development in the field of optical fiber sensing. When optical fiber is grated it changes the propagation of light within the fiber core. These gratings are produced due to perturbations caused by surrounding environment. The change in properties due to gratings has led to extensive study in fiber optic based sensor devices.

Fiber gratings can be broadly classified into two types. One is called as Fiber Bragg Gratings (FBG) and the other one is called as Long Period Grating (LPG). Fiber Bragg Gratings or Short period fiber gratings basically have very short period typically in the range of 10 to $100 \mu \mathrm{m}$. In FBG the forward propagating mode couples with the reverse counter propagating mode [1]. This particular coupling takes place at a specific wavelength which is called as Bragg wavelength. This Bragg wavelength is dependent on the period of FBG and the effective index of propagating mode. External conditions like temperature, strain, refractive index changes can affect these parameters which in turn will shift the Bragg wavelength. This forms the basis of FBG sensing. The FBG sensitivities to temperature and strain are $13 \mathrm{pm}$ $\mathrm{K}^{-1}$ and $1 \mathrm{pm} \mu \varepsilon^{-1}$ respectively [2].

In the case of Long Period Grating (LPG), the forward propagating mode couples with co propagating cladding modes. It has long periods ranging from $100 \mu \mathrm{m}$ to $1000 \mu$. These coupling show several dips in the transmission medium that is centred at discrete wavelengths. Each dip corresponds to coupling to different cladding mode.

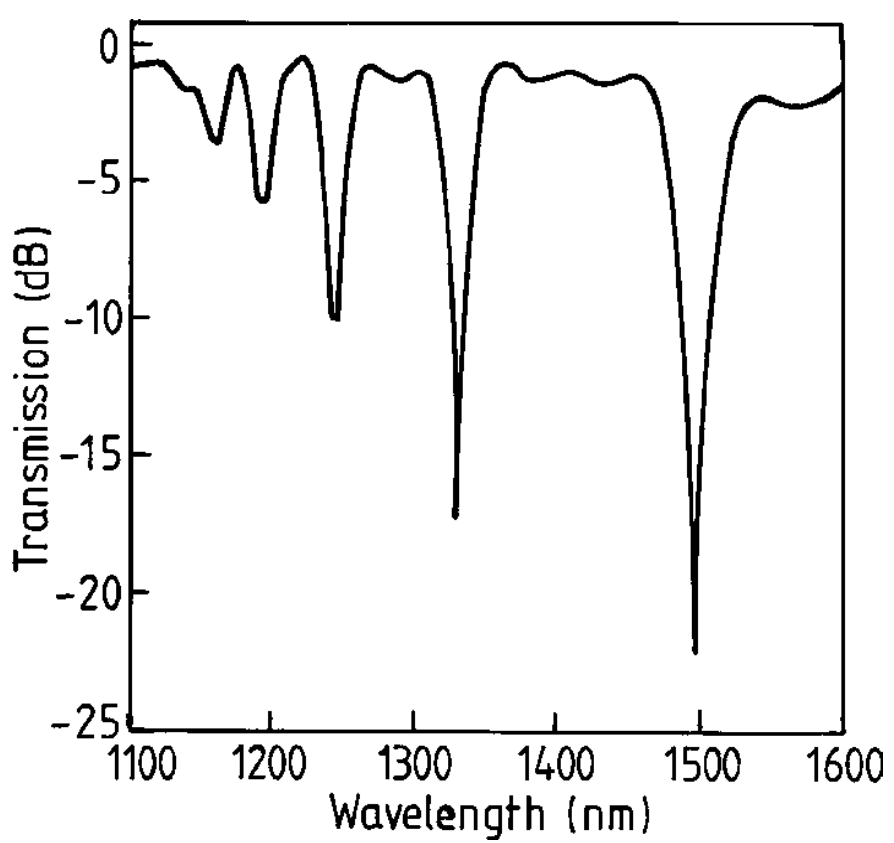

Fig -1 Transmission Spectrum of LPG fabricated on SMF28 with period $320 \mu \mathrm{m}$ [3]

Coupling to different wavelengths depends on phase matching condition given by $[3,4]$

$$
\lambda^{(\mathrm{m})}=\left(\mathrm{n}_{\text {core }}-\mathrm{n}_{\text {clad }}{ }^{(\mathrm{m})}\right) \Lambda
$$

Where $\mathrm{n}_{\text {core }}$ and $\mathrm{n}_{\text {clad }}$ is the effective index of core and cladding respectively. $\lambda^{(\mathrm{m})}$ is the coupling wavelength at $\mathrm{m}^{\text {th }}$ cladding mode. These coupling wavelengths is dependent on LPG period, length of the LPG and surrounding environment like temperature, strain, refractive index changes. Changes in these parameters can alter the phase matching conditions for coupling to different cladding modes and hence modifies the dips in the spectrum. 
The LPG sensitivity depends on composition of the fiber and the order of cladding mode and thus is different for each dip in the spectrum. These different responses make them an attractive choice for sensor applications including multi parameter sensor [4].

\section{LPG FABRICATION}

LPG on optical fiber can be fabricated by making periodic perturbation on the refractive index of core. This can be done by making permanent modifications on the RI of fiber or by making physical deformations of the fiber.

\subsection{LPG Fabrication using Ultraviolet (UV)}

\section{Irradiation}

$\mathrm{UV}$ radiation is the most common method to fabricate LPG on optical fiber. It is typically performed on Ge-doped silica fibers in the wavelength range of 193 to $266 \mathrm{~nm}$ [5]. This is again done in many ways. For example it can be done by a point by point basis or the entire length of LPG may be exposed to the radiation by using a patterned mirror [6] or by using an amplitude mask[7] or by using a microlens array[8].

An LPG fabrication using amplitude mask is shown.

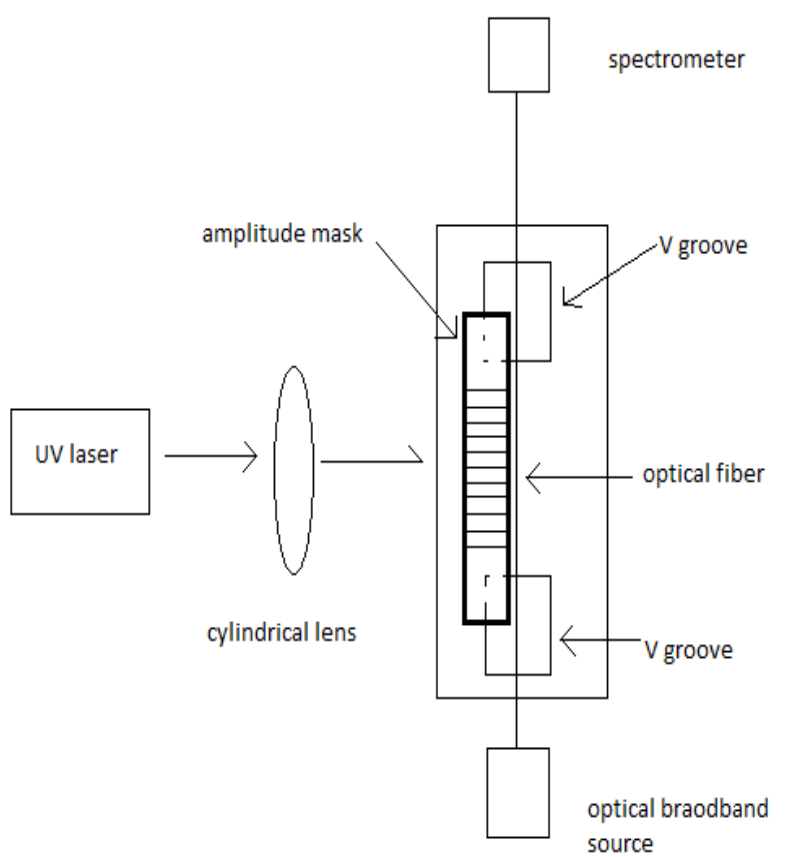

Fig -2 LPG fabrication using UV irradiation [17]

A broadband optical source is used to illuminate the optical fiber through amplitude mask of a particular period that is fabricated from either chrome plated silica [7] or a metal foil. The cylindrical lens produces a focused line along the axis of the fiber.

UV irradiation is widely used in FBGs [9]. This process improves the spectral characteristics and stability of LPG spectrum. Its useful in case of LPGs in non polarization maintaining fibers as it induced birefringence that produces polarization splitting in the dips seen in the transmission spectrum of LPG [10]. This method actually produces refractive index perturbation and during the process an unstable component is produced which changes the ventral wavelengths of the dips and the coupling strength as it decays with time [7]. Thermal annealing can be used to remove the unstable component but care must be taken for LPGs in certain applications. The photosensitivity of these fibers can be increased if the fibers are hydrogen loaded. It further shifts the central wavelengths and peak loss of the attenuation bands after fabrication as the hydrogen diffuses into the fiber [11].

The characteristics of LPG transmission spectrum can be further modified after the fabrication process by etching the fiber diameter. It changes the effective index of the cladding modes and increases the central wavelengths. It also brings about a change in the electric field profile of the cladding modes as it changes the overlap integral and the coupling coefficient which in turn changes the central wavelengths of the attenuation bands $[12,13]$.

\subsection{Irradiation of Femtosecond pulses in Infrared}

\section{Region}

LPGs can also be fabricated using focused irradiation of femtosecond pulses. This particular method causes a permanent change in the index [14]. It produces periodic perturbation of refractive index of core by making use of light hat cannot be absorbed by core and cladding glasses or any polymer coating. LPGs fabricated using this method in the near infrared region $(800 \mathrm{~nm})$ [14] shows a very stable transmission spectrum upto $500^{\circ} \mathrm{C}$. As compared to UV irradiation technique this method shows high resistance to thermal decay without making use of any other stabilization technique.

\subsection{Ion Implantation Technique}

This is another method for fabricating LPGs. This produces periodic perturbation of refractive index mainly by densification of silica glass using atomic collisions [15]. There are a few disadvantages. This process increases the cladding refractive index due to which the transmission spectrum shows more losses as compared to other methods. Also it requires a special equipment to carry out the process. The main advantage of using this method is that it can be applied to any type of silica fibers without any prior photo sensitization.

\subsection{LPG Fabrication using CO2 Laser}

Periodic perturbations can also be induced by means of $\mathrm{CO}_{2}$ laser. This technique does not necessarily require hydrogen loaded fiber. LPGs fabricated using $10.6 \mu \mathrm{m}$ laser light was shown to have very high thermal stability [16]. This laser light was focused on a spot by means of lenses and LPG is fabricated using periodical local heating. This method is considered to be flexible than UV irradiation. 


\subsection{LPG Fabrication using Electric arc Discharge}

Electric arc discharge is yet another flexible, easy to use and low cost technique to fabricate LPG as compared to UV irradiation. Like $\mathrm{CO}_{2}$ laser this method too does not necessarily require hydrogen loaded fibers. An optical fiber is placed between the electrodes of a fusion splicing machine and exposing the fiber to electric arc. A load is placed on one end of the fiber to maintain a longitudinal stress and the other end is connected to a computer controlled translational stage. An electric current of about 8.5 to $10 \mathrm{~mA}$ for 0.5 to 2 seconds is applied on the fiber. The translational stage moves along the fiber after each discharge that is equal to the period [18]. LPGs fabricated by this method has shown greater thermal stability of transmission spectrum till $800^{\circ} \mathrm{C}$ [19] and if proper annealing conditions are applied then it can show thermal stability till $1190^{\circ} \mathrm{C}[20]$.

\section{THEORY OF LPG}

An LPG consists of high index core which is surrounded by a lower index cladding, surrounded by air. The coupling of forward propagating guided mode to cladding modes depends on the phase matching condition given by equation 1. The minimum transmission of the attenuation bands is given by [1]

$$
\mathrm{T}=1-\sin ^{2}(\kappa \mathrm{L})
$$

where $\mathrm{L}$ is the length of LPG, $\kappa$ is called the coupling coefficient.

The coupling coefficient is determined by the overlap integral of core and cladding and by the amplitude of propagation constants. The cladding generally has a large radius so therefore it supports many cladding modes. Efficient coupling is only possible in case of a large overlap integral. In order to have a large overlap integral the modes must have similar electric field profile [21]. Hence efficient coupling takes place in case of core and circularly symmetric cladding modes of odd order. The electric field profile of these cladding modes has peak amplitude located within the core whereas electric field profile of cladding modes of even order has very low amplitude in the core [21].

The first step towards analytical designing of LPG is to calculate the refractive index of core and cladding to determine the proper central wavelengths of the attenuation bands. The refractive index of core is generally calculated using the weakly guided field approximation of Gloge [22]. Likewise calculation of refractive index of cladding modes is presented in [21]. The effective indices and electric field mode profile of cladding modes depends on the core which must be considered for accurate characteristics of LPG [21]. The second step towards analytical designing of LPG requires the calculation of coupling coefficient which requires determination of electric field profiles of the modes.
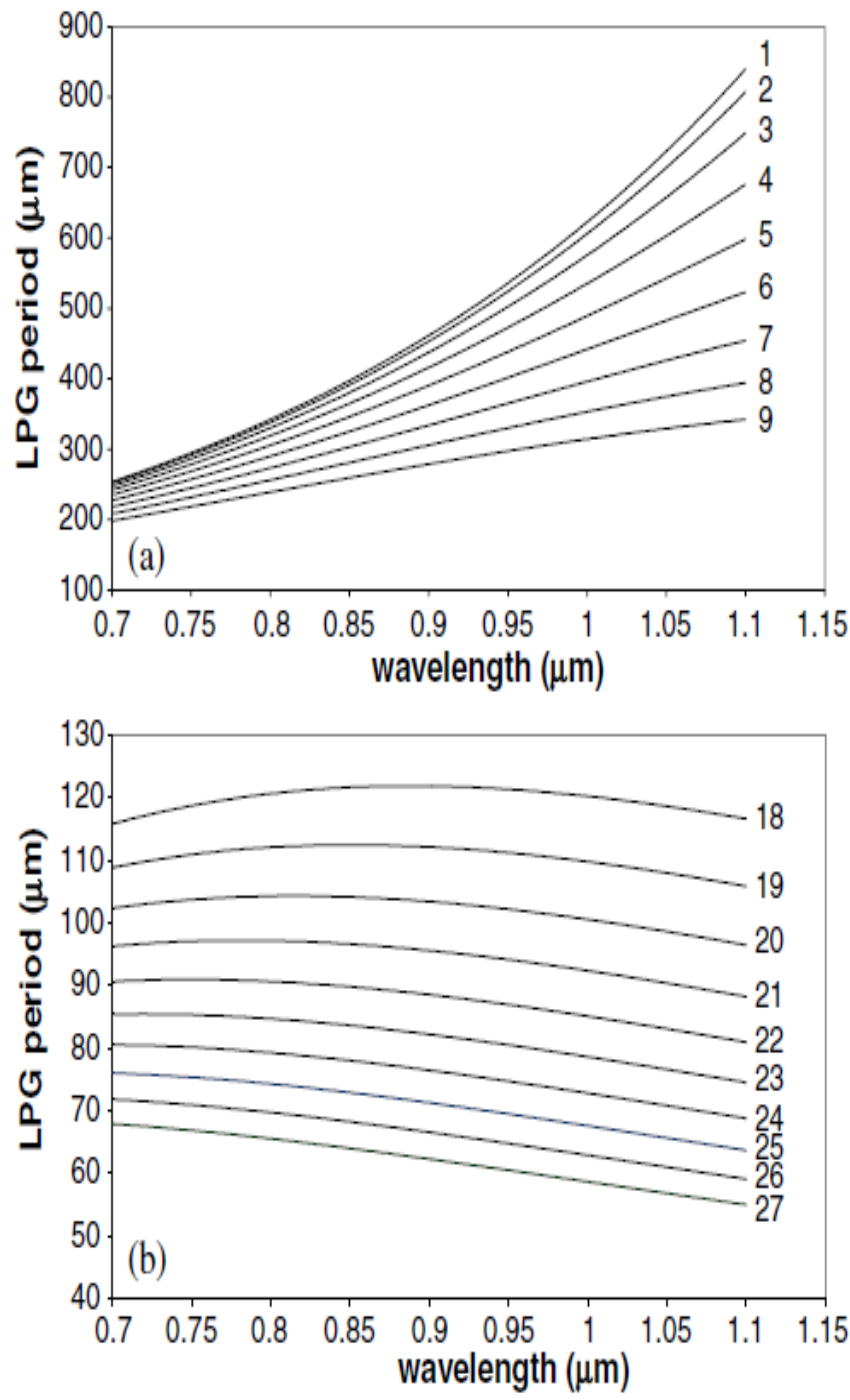

Fig -3. Coupling of guided mode with cladding modes of order (a) 1-9 (b) 18-27.[17]

Figure 3 was plotted using the method specified in [23]. It shows that coupling wavelength is dependent on the LPG period. These graphs shows that coupling to lower order modes take place at longer periods whereas coupling to higher order modes takes place at shorter periods. Figure $3 \mathrm{~b}$ also shows that in case of higher order modes two attenuation bands are produced if coupling to one cladding can take place at two wavelengths [24]. It also shows in case of mode order 18 that coupling conditions can bring about a change in the coupling efficiency but not in the wavelength [25].

\section{TEMPERATURE SENSITIVITY OF LPG}

The sensitivity of LPG towards temperature depends on three factors - period of LPG [4], order of the cladding mode [4] and composition of optical fiber [26]. When all these factors combine together we can produce an LPG sensor that can give positive temperature sensitivity, negative temperature sensitivity and temperature insensitive sensor as well. The temperature sensitivity can be given by taking chain rule derivative of equation (1) 
Equation (2):-[17]

$$
\frac{d \lambda}{d T}=\frac{d \lambda}{d\left(\delta n_{e f f}\right)}\left(\frac{d n_{e f f}}{d T}-\frac{d n_{c l}}{d T}\right)+\Lambda \frac{d \lambda}{d \Lambda} \frac{1}{L} \frac{d L}{d T}
$$

Where $\mathrm{T}$ is the temperature, $\lambda$ is the central wavelength of attenuation band, $n_{e f f}$ is the effective index of core, $n_{c l}$ is the effective index of cladding, $\delta n_{e f f}=n_{e f f}-n_{c l}$, L is the length of LPG, $\Lambda$ is period of LPG

The right hand side of the equation (3) which is given by change in differential refractive indices of core and cladding is the material contribution. This contribution depends strongly on the order of the cladding mode in which coupling takes place and the composition of the fiber. In case of lower order coupling modes the material contribution dominates whereas in case of higher order coupling modes the material effect is almost negligible [3]. The left hand side of equation (3) which is given by change in differential period of LPG is called by waveguide contribution. The sign of this term depends on the order of cladding mode. In case of lower order cladding modes the curve is positive and in case of higher order cladding modes the curve is negative from figure 3 (a) and (b). Thus by correct choice of period of LPG these two contributions can be balanced in such a way so that positive, negative and temperature insensitive LPG sensor can be obtained. It is also shown in [27] that the thermo optic coefficient which can be varied by the composition of fiber plays a strong role in temperature sensitivity of LPG. The graph shown in figure 4 is linear in room temperature [4] and non nonlinear for temperatures below $77 \mathrm{~K}$ [28].
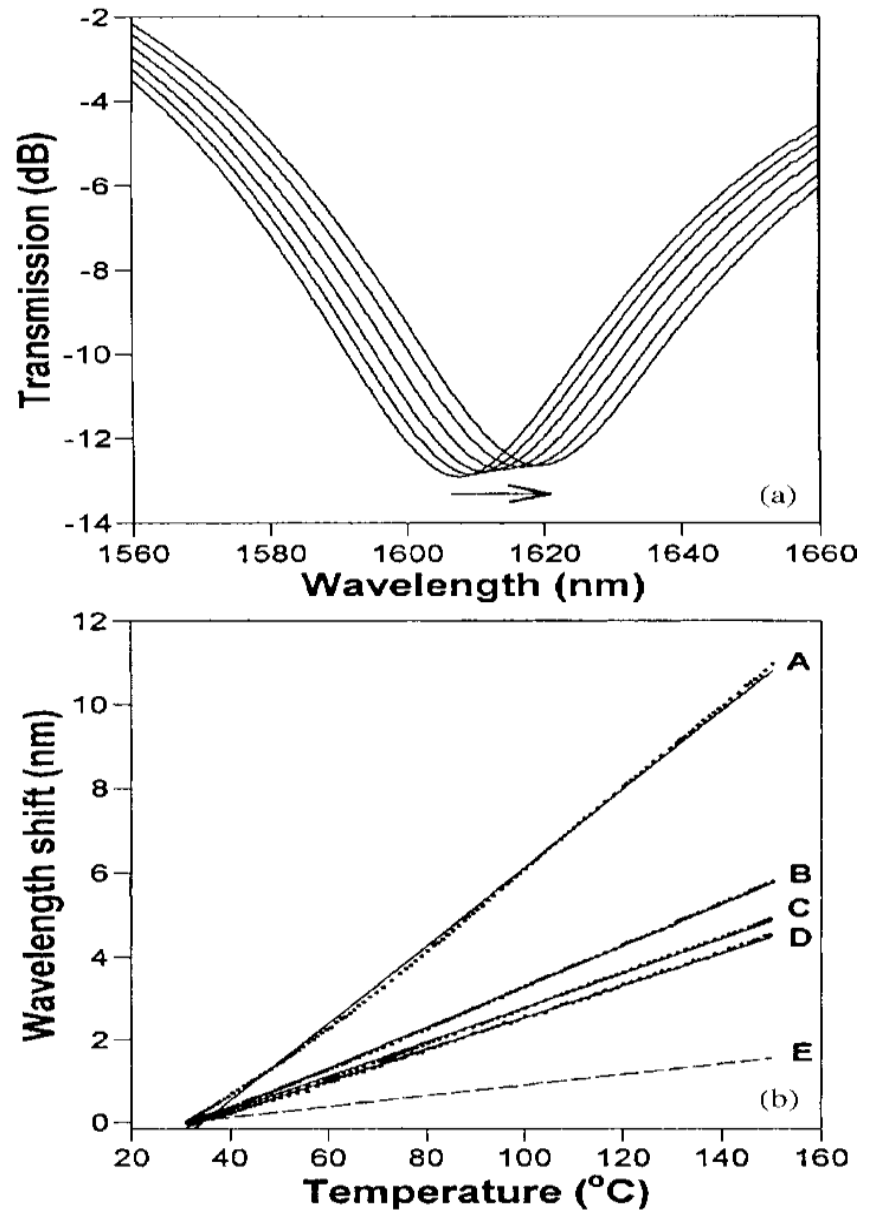

Fig - 4 (a) Wavelength shifts of LPG due to temperature. The shifts correspond to temperatures of 22.7, 49.1, 74.0, 100.29, 127.3, 149.7 $7^{0}$ LPG fabricated on Corning SMF-28 fiber having period $280 \mu \mathrm{m}$ [4]. (b) Shifts in wavelengths of attenuation bands A-D w.r.t. temperature. The dashed line shows wavelength shifts of FBG at $1550 \mathrm{~nm}$ [4].

LPGs fabricated in standard optical fiber shows temperature sensitivities from $3 \mathrm{~nm} / 100{ }^{\circ} \mathrm{C}$ to $10 \mathrm{~nm} / 100{ }^{\circ} \mathrm{C}$ [4]. This is an order larger than the FBG sensitivity. One can achieve lesser sensitivity by using LPGs having periods less than $100 \mu \mathrm{m}$ because in this case the coupling takes place at higher cladding modes where material contribution plays is very less. For example, LPGs having periods less than 40 $\mu \mathrm{m}$ shows sensitivity of about $1.8 \mathrm{pm}^{0} \mathrm{C}^{-1}$ which is less than that of FBG [3].

LPGs fabricated in photosensitive B- Ge doped show much larger sensitivity of about $275 \mathrm{~nm} / 100^{\circ} \mathrm{C}$ if proper mode order and coupling wavelength is chosen [29]. It has also been shown that if the fibers are surrounded by a material having large thermo optic coefficient the sensitivity is enhanced. These LPGs respond to both temperature and temperature induced refractive index changes as well [3032].

Temperature insensitive LPG can also be obtained by using athermal packaging [3]. This is achieved by bonding the fiber with a substrate like aluminium cylinder having a thermal expansion coefficient which induces a strain on the 
fiber. This strain causes wavelength shifts opposite to that induced by temperature. This showed about $45 \%$ decrease in temperature sensitivity [33]. Coating the fiber with a negative thermal optic coefficient also decreases the sensitivity to $0.07 \mathrm{~nm} / 100{ }^{0} \mathrm{C}$ [34]. Bend sensitivity also plays a role in reduction in temperature sensitivity [35].

\section{STRAIN SENSITIVITY OF LPG}

Strain sensitivity of LPG to axial strain is given by taking chain rule derivative of equation (1)

Equation (3):-[17]

$$
\frac{d \lambda}{d \varepsilon}=\frac{d \lambda}{d\left(\delta n_{e f f}\right)}\left(\frac{d n_{e f f}}{d \varepsilon}-\frac{d n_{c l}}{d \varepsilon}\right)+\Lambda \frac{d \lambda}{d \Lambda}
$$

The first term is the material contribution brought about by change in refractive index of core and cladding and strain optic coefficient. The second term is the waveguide contribution brought about by the slope $\mathrm{d} \lambda / \mathrm{d} \Lambda$. For LPGs having more than $100 \mu \mathrm{m}$ periods, the material contribution is negative and waveguide contribution is positive. Thus by appropriate choice of periods one can achieve positive, negative and zero sensitivity as well [3].

An LPG fabricated on Corning Flexcore having period of $340 \mu \mathrm{m}$ showed sensitivity of about $0.04 \mathrm{pm} \mu \varepsilon^{-1}$ [3] which is less than FBG at $1300 \mathrm{~nm}$. LPG having much lesser period of $40 \mu \mathrm{m}$ showed much larger sensitivity of $-2.2 \mathrm{pm}$ $\mu \varepsilon^{-1}$. The negative sensitivity is due to negative material and waveguide contributions [3]. Figure 5 shows strain sensitivity of LPG fabricated on Corning SMF-28 having period $280 \mu \mathrm{m}$ [4]. The bands were found to be at 1627.8 $\mathrm{nm}$ (A), $1315.9 \mathrm{~nm}$ (B), $1197.8 \mathrm{~nm}$ (C), 1128.3nm (D). The wavelength shift in band A was found to be non linear due to non linear waveguide contribution. The dotted line shows wavelength shifts of FBG at $1550 \mathrm{~nm}$ [4].

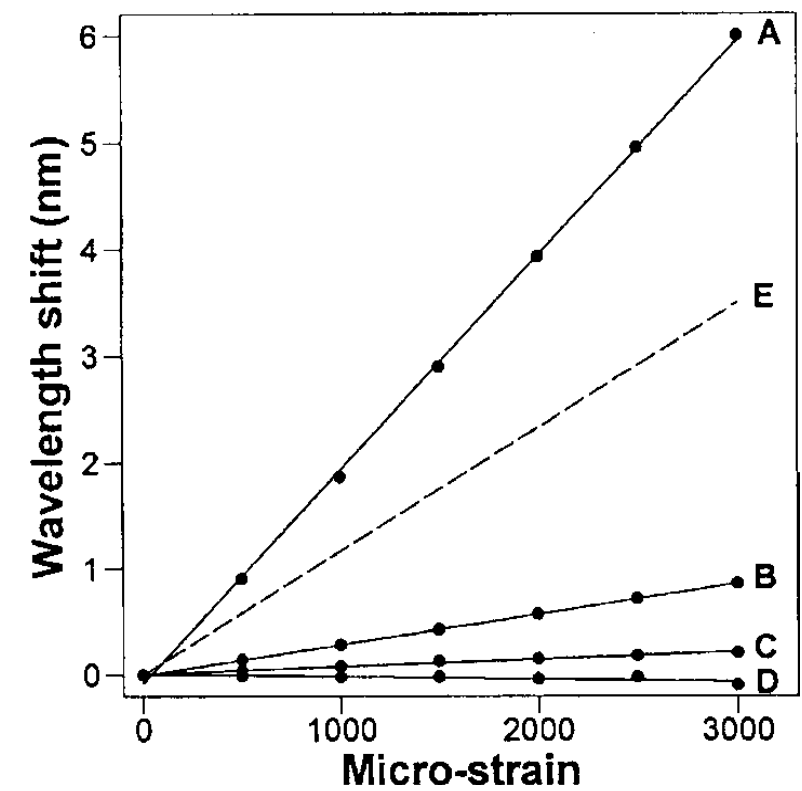

Fig -5 Wavelength shift of LPG fabricated on Corning SMF-28. [4]
When the period is made less than $100 \mu \mathrm{m}$ then the LPG becomes temperature insensitive and if the period is made more than $100 \mu \mathrm{m}$ then the LPG becomes strain insensitive. This enables the LPG to be used for measuring any one measurands since these periodicities correspond to regions where LPG can show highest sensitivity to strain and highest sensitivity to temperature. Moreover the dependence of wavelength shifts on coupling to cladding mode order can also be exploited to separate out the strain and temperature responses or measure both the parameters simultaneously [36].

The effect on strain sensitivity when transverse load is applied on LPG is checked in [37, 38]. When a transverse load is applied on LPG then it induces a birefringence and the attenuation bands are split into two. The wavelengths separation increases with the applied load and this lead to increased sensitivity of about $500 \mathrm{~nm} \mathrm{~kg}^{-1} \mathrm{~mm}^{-1}$ which is 800 times greater than fiber bragg grating [38].

\section{CONCLUSION}

The sensitivity of LPG to temperature and strain depends on the LPG period, the mode order in which the coupling takes place and the fiber composition. Greater temperature sensitivity can be obtained by choosing period more than $100 \mu \mathrm{m}$. Techniques to achieve enhanced temperature sensitivity and reduced temperature sensitivity is discussed. Higher strain sensitivities can be obtained by choosing LPG periods less than $100 \mu \mathrm{m}$. Application of transverse load on LPG has shown increased strain sensitivity as compared to FBG. The period, mode order and the features of transmission spectrum shows the ability of LPG to show simultaneous and independent responses of temperature and strain. The properties of LPG and its various fabrication techniques are also outlined.

\section{REFERENCES}

[1]. Kashyap R 1999 "Fibre Bragg Gratings" (NewYork: Academic)

[2]. Kersey A D, Davis M A, Patrick H J, LeBlanc M, Koo K P, Askins C G, Putnam M A and Friebele E J 1997 "Fiber Grating Sensors" J. Lightwave Technol. 15 1442-63.

[3]. Bhatia V, Campbell D K, Sherr D, D'Alberto T G, Zabaronick N A, Ten Eyck G A, Murphy K A and Claus R $\mathrm{O}$ "Temperature insensitive and strain insensitive long period grating sensors for smart structures" Opt. Eng. 1997 36 1872-6.

[4]. Bhatia V "Applications of long period grating to single and multi-parameter sensing" 1999 Opt. Express 4 457-66

[5]. Kashyap R "Fibre Bragg Gratings" (London: Academic) 1999

[6]. Patrick H J, Askins C G, Mcelhanon R W and Friebele E J "Amplitude mask patterned on an excimer laser mirror for high intensity writing of long period gratings" Electron. Lett. 199733 1167-8.

[7]. Vengsarkar A M, Lemaire P J, Judkins J B, Bhatia V, Erdogan $\mathrm{T}$ and Sipe J E "Long period fibre gratings as band rejection filters" J. Lightwave Technology 199714 58-64. 
[8]. Lu S Y, Tan H Y and Demokan M S "Low cost microlens array for long period grating fabrication" Electron Lett. 199935 79-80

[9]. Bennion I, Williams J A R, Zhang L, Sugden K and Doran N "UV-written in-fibre Bragg gratings" Opt. Quantum Electron 199628 93-135.

[10]. Duhem O and Douay M "Effect of UV-induced birefringence on long-period grating coupling characteristics" Electron. Lett. 200036 416-17

[11]. Guan B-Ou, Tam H-Y, Ho S-L, Liu S-Y and Dong X$\mathrm{Y}$ "Growth of long-period gratings in H2-loaded fibre after 193 nm UV inscription" IEEE Photon. Technol. Lett. 2000 12 642-4

[12]. Vasiliev S A, Dianov E M, Varelas D, Limberger H G and Salathe R P "Post-fabrication resonance peak positioning of long period cladding-mode-coupled gratings" Opt. Lett. 199621 1830-2.

[13]. Zhou K, Liu H and HU X "Tuning the resonant wavelength of long period fibre gratings by etching the fibre's cladding" Opt. Commun. 2001197 295-9

[14]. Kondo, Y.; Nouchi, K.; Mitsuyu, T.; Watanabe, M. \& Kazansky, P. "Fabrication of long-period fiber gratings by focused irradiation of infrared femtosecond laser pulses", Optics Letters, 1999 Vol. 24, pp. 646-648.

[15]. Fujimaki, M.; Nishihara, Y., Ohki, Y. ; Brebner, J. \& Roorda S.). "Ion-implantation induced densification in silica-based glass for fabrication of optical fiber gratings". Journal of Applied Physics, 2000 Vol.88, No.10, pp. 55345537, ISSN 0021-8979.

[16]. Davis D D, Gaylord T K, Glytsis E N and Mettler S C "CO2 laser induced long-period fibre gratings: spectral characteristic, cladding modes and polarisation independence" Electron. Lett. 199834 1416-17.

[17]. StephenWJames and Ralph P Tatam "Optical fibre long-period grating sensors: characteristics and application" Meas. Sci. Technol. 142003 R49-R61.

[18]. Catarina Silva, João M. P. Coelho, Paulo Caldas, and Pedro Jorge "Fibre Sensing System Based on Long-Period Gratings for Monitoring Aqueous Environments" Fiber Optic Sensors.

[19]. Humbert G and Malki A "Electric-arc-induced gratings in non-hydrogenated fibres: fabrication and hightemperature characterizations" J. Opt. A: Pure Appl. Opt. 20024 194-8

[20]. Rego G, Okhotnikov O, Dianov E and Sulimov V "High-temperature stability of long-period fibre gratings using an electric arc" J. Lightwave Technol. 200119 1574-9 [21]. Erdogan T "Cladding mode resonances in short and long period fibre grating filters" J. Opt. Soc. Am. 199714 1760-73 Erdogan T "Cladding mode resonances in short and long period fibre grating filters: errata" J. Opt. Soc. Am. 2000172113.

[22]. Gloge D "Weakly guiding fibres" Appl. Opt. 197110 2252-8.

[23]. Patrick H J, Kersey A D and Bucholtz F “Analysis of the response of long period fibre gratings to external index of refraction" J. Lightwave Technol. 199816 1606-42.

[24]. Han Y G, Lee B H, Han W T, Paek U C and Chung Y "Resonance peak shift and dual peak separation of long period fibre gratings for sensing applications" IEEE Photon. Technol. Lett. 200113 699-701.

[25]. Grubsky V and Feinberg J "Long period fibre gratings with variable coupling for real-time sensing applications" Opt. Lett. 200025 203-5.

[26]. Shima K, Himeno K, Sakai T, Okude S and Wada A "A novel temperature-insensitive long-period grating using a boron-codoped germanosilicate core-fibre" Tech. Dig. OFC'1997 pp 347-8.

[27]. $\mathrm{Ng} \mathrm{M} \mathrm{N}$ and Chiang K S "Thermal effects on the transmission spectra of long-period fiber gratings" Opt. Commun. 2002208 321-7.

[28]. James S W, Tatam R P, Bateman R, Twin A and Noonan $\mathrm{P}$ "Cryogenic temperature response of fibre optic long period gratings" Electron. Lett. 2003 submitted.

[29]. Shu X, Allsop T, Gwandu B, Zhang L and Bennion I "High temperature sensitivity of long-period gratings in BGe codoped fibre" IEEE Photon. Technol. Lett. 200113 818-20.

[30]. Yin S, Chung K-W and Zhu X "A highly sensitivity long period grating based tuneable filter using a unique double cladding layer structure" Opt. Commun. $2001 \mathbf{1 8 8}$ 301

[31]. Khaliq S, James S W and Tatam R P "Enhanced sensitivity fibre optic long period grating temperature sensor" Meas. Sci. Technol. 200213 792-5

[32]. Abramov A A, Hale A, Windeler R S and Strasser T A "Widely tunable long-period fibre gratings" Electron. Lett. 199935 81-2

[33]. Qin L, Wei Z X, Wang Q Y, Li H P, ZhengW, Zhang Y S and Gao D S "Compact temperature compensating package for long period fibre gratings" Opt. Met. 200014 239-42.

[34]. Jang J N, Kim S Y, Kim S W and Kim M S "Temperature insensitive long-period fibre gratings" Electron. Lett. 1999352134.

[35]. Ng M N, Chen Z H and Chiang K S "Temperature compensation of long-period fiber grating for refractiveindex sensing with bending effect" IEEE PhotonTechnol. Lett. 200214 361-2.

[36]. Bhatia V, Campbell D, Claus R O and Vengsarkar A $\mathrm{M}$ "Simultaneous strain and temperature measurement with long-period gratings" Opt. Lett. 199722 648-50.

[37]. Liu Y, Zhang L and Bennion I "Fibre optic load sensors with high transverse strain sensitivity based on longperiod gratings in B/Ge co-doped fibre" Electron. Lett. 1999 35 661-2.

[38]. Zhang L, Liu Y, Williams J A R and Bennion I “ Design and realisation of long-period grating devices in conventional and high birefringence fibers and their novel applications as fibre optic load sensors" IEEE J. Sel. Top. Quantum Electron. 19995 1371-8. 


\section{BIOGRAPHIES}

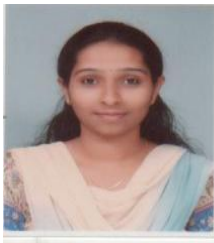

Liji Sreenivasan Madhavan has completed her Bachelor of Engineering in Electronics from Pillai's Institute of Information Technology, Panvel, India. She is currently working as Assistant Professor in Saraswati College of engineering, Kharghar, India.

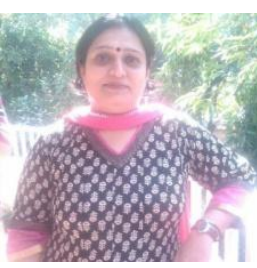

Chembur, India.
Prof. Manisha Chattopadhyay has completed Bachelor of Engineering and Master of Engineering in Electronics and Telecommunication. She is currently working as Associate Professor in Vivekanand Education Society's Institute of Technology, 